\title{
Variabilidad en la explotación y distribución de obsidianas en la Puna Meridional argentina
}

Patricia S. Escola ${ }^{1}$

\section{RESUMEN}

Se presenta la caracterización geoquímica de dos fuentes de aprovisionamiento de obsidiana localizadas en el Depto. Antofagasta de la Sierra, Catamarca. Esta información se combina con resultados de análisis de procedencia (INAA) realizados sobre artefactos arqueológicos de obsidiana de 20 sitios datados entre 2200-600 AP y ubicados en diversos sectores ambientales del Noroeste Argentino. Dado que los rangos de circulación resultantes para ambas fuentes presentan diferencias, este trabajo intenta iniciar una evaluación de la naturaleza diferencial del intercambio a partir de las estrategias de aprovisionamiento y manejo de las obsidianas de ambas fuentes consideradas. Se analizan entonces conjuntos líticos recuperados tanto de una de las fuentes de aprovisionamiento como del sitio Casa Chávez Montículos.

Palabras claves: fuentes de obsidiana - distribución diferencial - variabilidad en aprovisionamiento y consumo contextos ag ropastoriles - Puna Meridional argentina.

\begin{abstract}
Geochemical characterization of two obsidian sources located in the Department of Antofagasta de la Sierra (Catamarca) is presented. This information is combined with results of provenance analysis (INAA) carried out on obsidian archaeological artifacts from 20 sites dated between 2200-600 BP and located in several ecological zones of Northwest Argentina. Considering that the circulation range for the two sources are different, this paper attempts to evaluate the differential nature of, procurement, consumption and exchange strategies for both these obsidian sources. We further analyzed the lithic assemblages recovered from one of the obsidian sources and from the Casa Chavez Montículos site.
\end{abstract}

Key words: obsidian sources - differential distribution - variability in procurement and consumption - agropastoralist contexts - Southern Argentine Puna.

Recibido: abril 2004. Manuscrito revisado aceptado: octubre 2004.

1 CONICET - Universidad Nacional de Catamarca. $1^{\circ}$ de Mayo 1502. San Fernando del Valle de Catamarca (4700), Catamarca, ARGENTINA. Email: suyu@ arnet.com.ar

\section{Introducción}

Los estudios de procedencia de recursos líticos han permitido obtener evidencia confiable acerca del movimiento o transporte de los mismos desde sus fuentes específicas hacia otras localidades geográficas. En este sentido, responden a una de las primeras interrogantes que se plantean frente a la presencia de materiales exóticos en los contextos arqueológicos. Esto ha permitido orientar diversas investig aciones hacia la identificación de redes de intercambio prehispánico. Sin embargo, es un hecho que los estudios geoquímicos por sí solos no pueden aportar información acerca de la producción que precede a la distribución como tampoco acerca del consumo de los artefactos líticos transportados. En este sentido, si se considera que el intercambio juega un rol clave en el desarrollo social y económico de los grupos humanos (Scarre 1993) es necesario avanzar en el conocimiento de su naturaleza así como de los mecanismos y estrategias que lo sustentan.

Ahora bien, diversos tipos de artefactos han sido identificados geoquímicamente en estudios de procedencia, sin embargo, aquellos elaborados en obsidiana resultan óptimos para la asignación de dicha procedencia. Las fuentes de obsidiana, desde el punto de vista de su composición química, son altamente homogéneas. Pero las diferencias composicionales entre distintas fuentes son, a su vez, tan significativas que pueden ser fácilmente detectadas por variados métodos analíticos. Asimismo, si bien los artefactos de obsidiana pueden encontrarse frecuentemente en una gran variedad de lugares y contextos arqueológicos, las fuentes de obsidiana son geográficamente limitadas. De este modo, cuando se recuperan artefactos de obsidiana alejados de sus fuentes de procedencia, la confiabilidad de la identificación de la fuente es elevada (Hughes 1998; Glascock 2002).

En este marco, este trabajo pretende comenzar a aportar información acerca de la dinámica de explotación, distribución y consumo de un recurso 
lítico como la obsidiana en contextos agropastoriles de la Puna Meridional argentina. Se presenta, en primera instancia, la caracterización geoquímica de dos fuentes de obsidiana localizadas en los sectores noroeste y sureste del Depto. Antofagasta de la Sierra. Dicha caracterización se basa en análisis de activación neutrónica efectuados sobre muestras geológicas. Esta información se combina con resultados de análisis de procedencia (INAA) realizados sobre artefactos arqueológicos de obsidiana de 20 sitios. Cabe destacar que, temporalmente, se contempla evidencia desde $2200 \mathrm{AP}$ hasta $600 \mathrm{AP}$, mientras que espacialmente, estos sitios se encuentran ubicados en diversos sectores ambientales del Noroeste Argentino. Dado que los rangos de circulación resultantes para ambas fuentes presentan diferencias, se considera importante, en segunda instancia, comenzar a evaluar la naturaleza diferencial del intercambio a partir de las estrategias de aprovisionamiento y consumo del recurso lítico. Para ello, se toman en consideración conjuntos líticos recuperados tanto de una de las fuentes de obsidiana como del sitio arqueológico Casa Chávez Montículos (Antofagasta de la Sierra) que presenta evidencias de uso de ambas obsidianas.

\section{Fuentes y artefactos de obsidiana: Identificación y procedencia}

Mi acercamiento a la obsidiana surge a partir de investig aciones arqueológicas desarrolladas en el Depto. Antofagasta de la Sierra y orientadas al estudio del uso del espacio y manejo de recursos líticos en contextos agropastoriles comprendidos entre 2200 AP y 600 AP (Figura 1).

Al respecto, estudios tecnológicos efectuados sobre muestras artefactuales de dos sitios estratificados CChM1 (Casa Chávez Montículos (Montículo 1), en el fondo de cuenca, y RG1 (Real Grande-1) en el sector microambiental de quebradas de altura indicaban que estos grupos agropastoriles manejaban un variado conjunto de materias primas de calidad diversa, destacándose entre éstas la obsidiana en su carácter de recurso no local o exótico. Lo interesante de este recurso es que, a pesar de su no localidad, su frecuencia de aparición en los registros es relevante. Sobre un total de 12 materias primas identificadas en los artefactos analizados, y tomando en consideración la producción total (instrumentos, núcleos y desechos de talla), la obsidiana sobresale en
CChM1 con un porcentaje de $30.0 \%(n=1524)$ mientras que en RG1 muestra una frecuencia de $16.9 \%(\mathrm{n}=2497)$ (Escola 2000).

Esto llevó a plantearme preguntas acerca de su procedencia. De este modo, se iniciaron a fines de 1989 tareas tendientes a la localización de fuentes de aprovisionamiento de obsidiana. Luego, estos estudios se vieron completados en 1997 con la puesta en marcha, bajo una perspectiva más regional e integradora abarcando todo el Noroeste Argentino, de un programa de investigación orientado no sólo a la localización y caracterización geoquímica de fuentes de obsidiana, sino también a la determinación de procedencia de muestras arqueológicas de diversos sitios de la región. Como resultado de estas actividades, en primer término, se lograron localizar y caracterizar 10 fuentes de obsidiana. Específicamente, en lo que respecta al sector argentino de la Puna Meridional o Puna Salada (Santoro y Núñez 1987), se identificaron cuatro fuentes de aprovisionamiento (OnaLas Cuevas, Cueros de Purulla, Chascón y Valle Ancho), de las cuales las tres primeras se encuentran localizadas en el Departamento de Antofagasta de la Sierra. De manera general, se puede decir que se trata de cuerpos y coladas dómicas asociadas a grandes calderas y estratovolcanes del Terciario y eventos aislados del Cuaternario (Viramonte et al. 1988; González 1992). Cabe destacar que, a los fines de este trabajo, se tratarán solamente las dos fuentes de mayor relevancia en la región.

La primera de dichas fuentes, y la más intensamente estudiada, es la fuente Ona ubicada a $3700 \mathrm{~m} . \mathrm{snm}$, y a alrededor de $80-90 \mathrm{~km}$ de la localidad de Antofagasta de la Sierra (Figura 1). Los relevamientos realizados en dicha fuente indican la existencia de varios afloramientos (Sectores 1, 2, 3 y 4), así como también sectores con gran densidad de nódulos (Sectores A y B y pendientes de los Sectores 1 y 3) (Figura 2), de tamaños variados, entre los cuales los de mayor diámetro no superan los $30 \mathrm{~cm}$. Según los distintos sectores, el vidrio (translúcido) muestra variaciones de coloración que incluyen el negro, gris-negro, gris, plateado y marrón rojizo. En las adyacencias de la fuente se observa una gran dispersión de rodados de obsidiana, de variado tamaño, producto de la gravedad y de otros procesos geológicos que involucran el transporte de material primario. A su vez, $2 \mathrm{~km}$ al sur de la fuente Ona, en la 


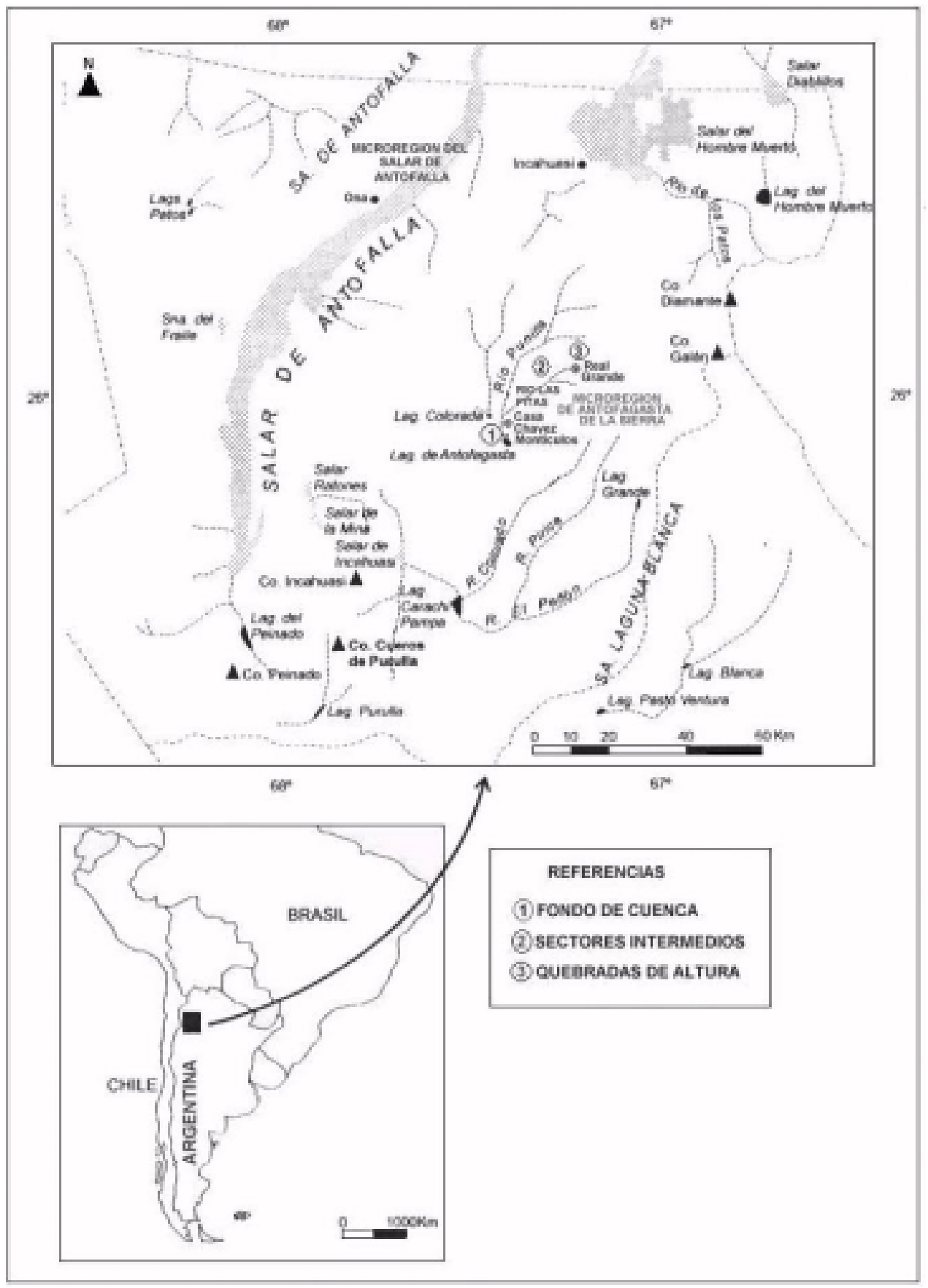

Figura 1. Localización de sitios arqueológicos: Casa Chávez Montículos y fuentes de obsidiana. Mapa tomado y adaptado de Aschero y colaboradores (2002).

quebrada de Las Cuevas y a 3900 m.snm, se localizó una fuente de obsidiana (Las Cuevas) aún más discreta que la anterior. $\mathrm{Al}$ respecto, se puede destacar que el rango de tamaños de los nódulos como también la variedad de coloración de la materia prima es similar a la encontrada en Ona. En función de la realización de análisis geoquímicos se recolectaron diez muestras de obsidiana de la fuente de Ona y una muestra piloto correspondiente al afloramiento de Las Cuevas 


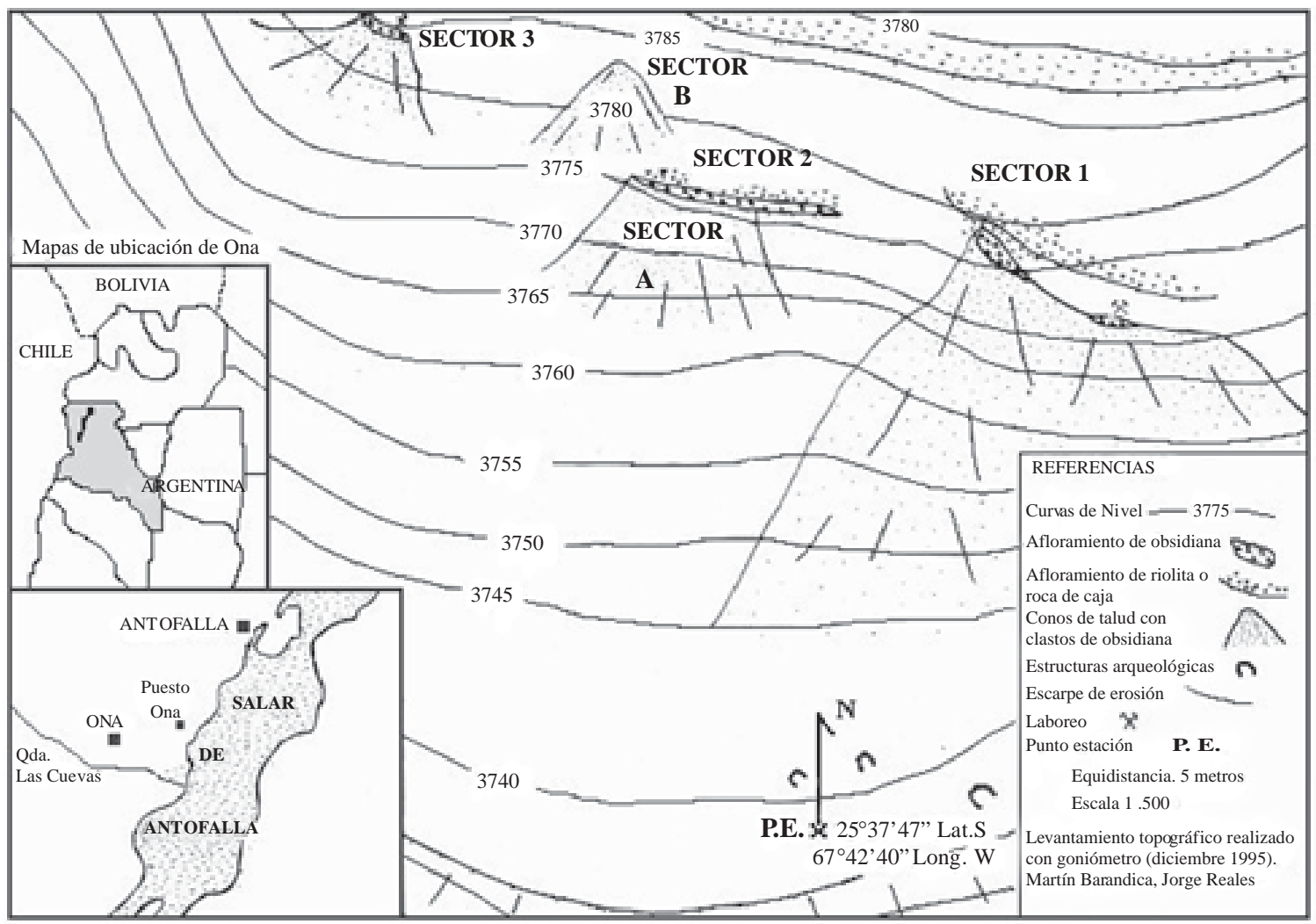

Figura 2. Fuente Ona: Sectores y estructuras relevadas.

atendiendo a la posibilidad que correspondiera al mismo magma que Ona. Cabe destacar que los especímenes recogidos en Ona procedían de los distintos afloramientos relevados (Sectores 1 a 4), así como también de los sectores de nódulos en pendiente (Sectores A y B).

Durante los relevamientos efectuados en Ona se registraron también evidencias de producción lítica vinculadas a la explotación de este recurso. La concentración de artefactos adscribibles a actividades de reducción primaria abunda en distintos sectores de la fuente. En el área de acceso al Sector 1 (Figura 2), prácticamente al pie de la pendiente, se ubican tres estructuras de forma semicircular, de pared simple, sin argamasa, con bloques no canteados colocados en forma superpuesta, a provechando en algunos casos la presencia de grandes bloques in situ. Se han registrado, en clara asociación con estas estructuras, episodios de talla lítica. Asimismo, ya en el Sector 1, a una distancia de aproximadamente $20 \mathrm{~m}$ del afloramiento, se encuentra una explanada que cubre una extensión de $20 \mathrm{~m}^{2}$, en cuya superficie se ha documentado la presencia de percutores, núcleos y lascas externas. Eventos similares han sido observados también en el Sector 3. Si bien el material arqueológico recuperado se encuentra aún en proceso de análisis, las observaciones de campo realizadas hacen referencia en términos generales a evidencias de testeo de nódulos, descortezamiento primario y preparación de núcleos, así como también extracción de formas base (lascas) de tamaños grandes y muy grandes.

Por su parte, la fuente Cueros de Purulla, a $4380 \mathrm{~m}$.snm, se encuentra ubicada a alrededor de $60-70 \mathrm{~km}$ al sureste de la localidad de Antofagasta de la Sierra. En este caso, la materia prima es de excelente calidad para la talla y aparece en forma de grandes bloques y nódulos, algunos excediendo los $25 \mathrm{~cm}$ de lado. En cuanto a la coloración, esta obsidiana de tipo opaca varía entre el negro, el gris oscuro y negro-rojizo. Para los fines de análisis geoquímicos posteriores se recolectaron 13 muestras de mano abarcando el lado norte y el lado sur del domo volcánico. Cabe destacar que, si bien no pudieron llevarse adelante relevamientos 
arqueológicos sistemáticos, pudieron detectarse en el campo diversos eventos de producción lítica.

Todas las muestras de obsidiana recolectadas en las fuentes antes mencionadas ( 24 en total) fueron sometidas a análisis de activación neutrónica (INAA) en dependencias del Missouri University Research Reactor (M.U.R.R.) utilizando los procedimientos detallados en trabajos previos (Cobean et al. 1991) para la medición de 27 elementos.

Si bien es cierto que sólo se ha analizado una muestra de la formación de la quebrada de Las Cuevas, en calidad de piloto, los resultados parecerían indicar que los afloramientos de Ona y Las Cuevas proceden de un mismo magma. Esta situación de flujos lávicos notoriamente discretos en el terreno pero con composiciones químicas similares ya ha sido discutida por algunos autores (Sidrys et al. 1976; Hurtado de Mendoza y Jester 1978; Hurtado de Mendoza 1986). De no mediar una caracterización geoquímica más afinada se propone un tratamiento más regional de estas fuentes, haciendo referencia a ellas como "área de fuentes" o "sistema de fuentes" bajo la denominación general de Ona (ver Yacobaccio et al. 2002 y 2004 para mayores detalles acerca del tipo químico identificado).

Ahora bien, la asignación de procedencia de artefactos de obsidiana a nivel regional requiere no sólo de la localización y caracterización de fuentes de obsidiana, sino también de la posibilidad de acceder a distintos registros arqueológicos. En este sentido, el programa de investigación ya mencionado procedió a la evaluación de procedencia de artefactos de obsidiana recuperados de 37 sitios arqueológicos datados entre 2200 AP y 400 AP (Yacobaccio et al. 2004). En función de los objetivos propuestos en este trabajo, voy a centrar mi atención en los resultados de procedencia obtenidos de 20 de estos sitios, algunos de ellos multicomponentes, ubicados en las provincias de Catamarca y Salta y dentro del lapso temporal considerado en esta investigación que se restringe del 2200 AP al 600 AP. Cabe destacar que, de estos 20 sitios arqueológicos considerados, se analizó un total de 126 especímenes de obsidiana. Al respecto, se debe señalar que no todos los sitios arqueológicos están representados con la misma proporción de artefactos. En algunos casos, sólo unas piezas pudieron ser analizadas mientras que en otros, como por ejemplo Casa Chávez Montículos (Montículos 1 y 4) (n=46) y Real Grande$1(\mathrm{n}=21)$, las muestras superan la veintena. Esto se debe en parte a la escasez de obsidiana encontrada en algunos sitios y, en parte también, a la disponibilidad de acceso de muestras de obsidiana procedentes de sitios analizados en el marco de investigaciones propias como vinculadas a otros proyectos de investigación. Asimismo, en cuanto al contexto de las muestras artefactuales cabe mencionar que, en su mayoría, corresponden a contextos excavados estratigráficamente, aunque en algunos pocos casos se incluyeron también muestras superficiales.

Como se puede apreciar en la Tabla 1, del total de sitios, cinco provienen de la provincia de Salta y los restantes 15 pertenecen a la provincia de Catamarca. Por su parte, en lo que respecta a su distribución ecológica, la muestra considerada incluye no sólo sitios de diversos sectores de la Puna sino también de los valles mesotérmicos orientales adyacentes y la selva montana.

Pero, ¿qué muestran los análisis de procedencia acerca de la circulación de obsidianas? En principio, se ha podido establecer de manera no ambigua que el $61.0 \%$ de los artefactos de los sitios arqueológicos muestreados provienen de la fuente Ona y el $14.0 \%$ de Cueros de Purulla. Parte del $25.0 \%$ restante, más precisamente el $11.0 \%$, muestra la circulación de obsidianas de otras fuentes ya localizadas como son Zapaleri, Alto Tocomar, Quirón y Valle Ancho. A su vez, el otro 14.0\% de los artefactos de esta muestra arqueológica ha permitido, en principio, la identificación de ocho posibles fuentes adicionales de las cuales aún se desconoce su ubicación geográfica. Me refiero a las denominadas "Fuentes Desconocidas A, B, C, E, F, G, H, K y M (ver Tabla 1). Cabe destacar que todas ellas proceden de sitios de la provincia de Catamarca.

Específicamente, en cuanto a la distribución geográfica que reg istran culturalmente las obsidianas de las fuentes Ona y Cueros de Purulla, la Figura 3 permite efectuar una evaluación de los resultados alcanzados. Por un lado, se puede advertir en el sector meridional del N.O.A. una importante esfera de distribución que se origina en la fuente Ona evidenciando aproximadamente un rango de circulación de $340 \mathrm{~km}$. Al respecto, la obsidiana de esta fuente no sólo comprende a los sitios de 


\begin{tabular}{|c|c|c|c|}
\hline $\begin{array}{l}\text { Grupo } \\
\text { cronológico }\end{array}$ & Nombre y $\mathbf{n}^{0}$ de sitio & Localización & $\begin{array}{l}\text { Fuentes utilizadas y } \\
\text { cantidad de muestras }\end{array}$ \\
\hline 2200-1800 AP & $\begin{array}{l}\text { 1. Las Garzas } \\
\text { 2. Las Cuevas } \\
\text { 3. Cardonal (sup.) } \\
\text { 4. Montículo } 1 \text { (Casa Chávez) } \\
\text { Componente Inferior }\end{array}$ & $\begin{array}{l}\text { Valle de Lerma, Salta } \\
\text { Quebrada del Toro, Salta } \\
\text { Valle del Cajón, Catamarca } \\
\text { Antofagasta de la Sierra, } \\
\text { Catamarca }\end{array}$ & $\begin{array}{l}\text { Zapaleri (3) } \\
\text { Ona, Zapaleri y Tocomar (3) } \\
\text { Ona, Cueros de Purulla y Fuentes } \\
\text { Desconocidas B y K (4) } \\
\text { Ona, Cueros de Purulla y Fuente } \\
\text { Desconocida A (16) }\end{array}$ \\
\hline 1800-1100 AP & $\begin{array}{l}\text { 5. Bañado Viejo (sup.) } \\
\text { 6. Montículo 1(Casa Chávez) } \\
\text { Componente Superior } \\
\text { 7. Montículo } 4 \text { (Casa Chávez) } \\
\text { 8. Loma Alta } \\
\text { 9. Ingenio Arenal (sup.) } \\
\text { 10. Ampascachi } \\
\text { 11. Potrero Grande } \\
\text { 12. Matancillas (sup.) }\end{array}$ & $\begin{array}{l}\text { Valle de Santa María, Catamarca } \\
\text { Antofagasta de la Sierra, } \\
\text { Catamarca } \\
\text { Antofagasta de la Sierra, } \\
\text { Catamarca } \\
\text { Falda occidental del Aconquija, } \\
\text { Catamarca } \\
\text { Falda occidental del Aconquija, } \\
\text { Catamarca } \\
\text { Valle de Lerma, Salta } \\
\text { Quebrada del Toro, Salta } \\
\text { San Antonio de los Cobres, Salta }\end{array}$ & $\begin{array}{l}\text { Ona (6) } \\
\text { Ona, Cueros de Purulla y Fuentes } \\
\text { Desconocidas A y B (14) } \\
\text { Ona, Cueros de Purulla y Fuentes } \\
\text { Desconocida A, B y H (16) } \\
\text { Ona y Fuente Desconocida M (13) } \\
\text { Ona (1) } \\
\text { Ona (1) } \\
\text { Zapaleri y Tocomar (3) } \\
\text { Zapaleri y Tocomar (3) }\end{array}$ \\
\hline $1100-550 \mathrm{AP}$ & $\begin{array}{l}\text { 13. La Hoyada-3 (sup.) } \\
\text { 14. Tesoro (sup.) } \\
\text { 15. Real Grande-1 } \\
\text { Componente Inferior } \\
\text { 16. Real Grande-1 } \\
\text { Componente Superior } \\
\text { 17. Real Grande-10 } \\
\text { 18. Vega Incahuasi (sup.) } \\
\text { 19. Vega Cueros de Purulla (sup.) } \\
\text { 20. Rincón Chico (Sitio 15) } \\
\text { 21. De Las Cruces } \\
\text { 22. San Francisco (sup.) } \\
\text { 23. Las Grutas-12 (sup.) }\end{array}$ & $\begin{array}{l}\text { Valle del Cajón, Catamarca } \\
\text { Falda occidental del Aconquija, } \\
\text { Catamarca } \\
\text { Antofagasta de la Sierra, } \\
\text { Catamarca } \\
\text { Antofagasta de la Sierra, } \\
\text { Catamarca } \\
\text { Antofagasta de la Sierra, } \\
\text { Catamarca } \\
\text { Antofagasta de la Sierra, } \\
\text { Catamarca } \\
\text { Antofagasta de la Sierra, } \\
\text { Catamarca } \\
\text { Valle de Santa María, Catamarca } \\
\text { Orán, Salta } \\
\text { Chaschuil, Catamarca } \\
\text { Chaschuil, Catamarca }\end{array}$ & $\begin{array}{l}\text { Ona (1) } \\
\text { Ona y Fuente Desconocida J (1) } \\
\text { Ona, Cueros de Purulla, Quirón y } \\
\text { Fuente Desconocida B (12) } \\
\text { Ona y Fuentes Desconocidas } \\
\text { E, F y G (9) } \\
\text { Ona, Cueros de Purulla y Fuente } \\
\text { Desconocida E (6) } \\
\text { Ona, Cueros de Purulla y Valle } \\
\text { Ancho (4) } \\
\text { Ona, Cueros de Purulla y Fuentes } \\
\text { Desconocidas A y C (5) } \\
\text { Ona (1) } \\
\text { Zapaleri (1) } \\
\text { Ona (1) } \\
\text { Cueros de Purulla (2) }\end{array}$ \\
\hline
\end{tabular}

Tabla 1. Sitios arqueológicos y fuentes de procedencia por grupo cronológico. Todas las muestras son de excavación a menos que se indique su origen superficial.

la puna de Catamarca $\left(n^{\circ} 4,6,7,15,16,17,18\right.$, $19,22)$ que la contiene, sino que también alcanza a sitios arqueológicos del valle del Cajón $\left(\mathrm{n}^{\circ} 3\right.$, 13), de la falda occidental del Aconquija $\left(n^{\circ} 8,9\right.$, 14) y del valle de Santa María $\left(n^{\circ} 5,20\right)$ (todos en la provincia de Catamarca). Incluso también llega a sitios de la provincia de Salta, tan lejos al oriente como el valle de Lerma $\left(n^{\circ} 10\right)$ y, hacia el norte, hasta la quebrada del Toro $\left(\mathrm{n}^{\circ} 2\right)$.
Por otro lado, en lo concerniente a la fuente Cueros de Purulla, a pesar de que tiene una distribución importante en términos de sitios que se abastecieron de ella, es evidente que proveyó de obsidiana a sitios ubicados, principalmente, en la puna de Catamarca $\left(n^{\circ} 4,6,7,15,16,17,18\right.$, 23), observándose un único caso aislado en el valle del Cajón ( $\left.n^{\circ} 3\right)$. En este sentido, se podría decir que ésta sería una fuente menor funcionando de 


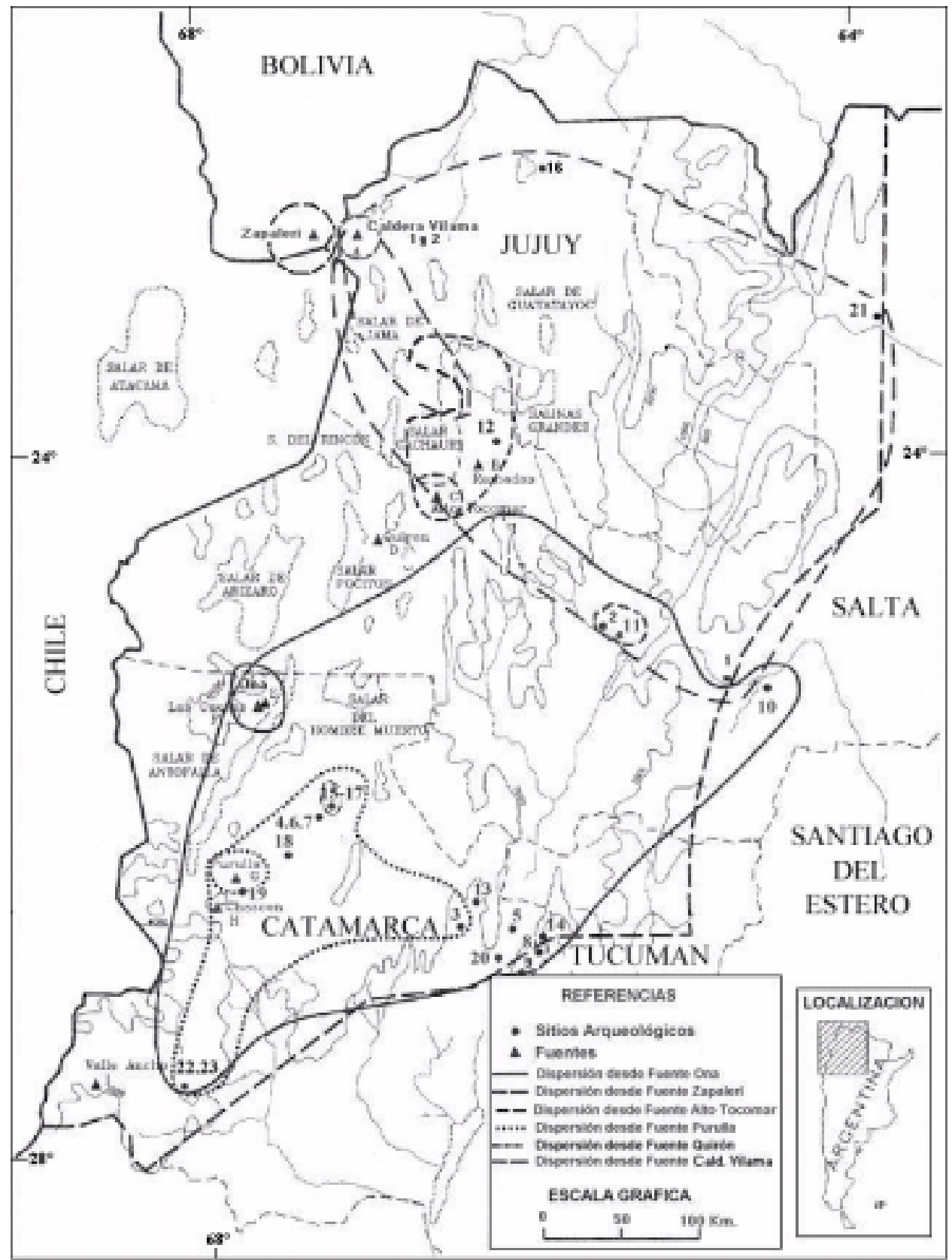

Figura 3. Mapa que muestra sitios arqueológicos y esferas de distribución por fuente de obsidiana. Tomado y adaptado de Yacobaccio y colaboradores (2004).

manera paralela a Ona. Es muy posible que la esfera de distribución de esta fuente se encuentre subrepresentada, pues se trata de una materia prima que no es reconocida macroscópicamente por los investigadores de diferentes proyectos como obsidiana, dada su opacidad, sino como basalto o sílice.

\section{Intercambio diferencial: Aprovisionamiento y consumo}

A través de los estudios de procedencia efectuados se ha podido advertir que, desde el punto de vista de su distribución geográfica, existen diferencias en los rangos de circulación y uso de las 
obsidianas de las fuentes tratadas. En función de comenzar a evaluar la naturaleza diferencial de estos desplazamientos de obsidiana, considero relevante analizar inicialmente información tecnológica, sobre los diversos contextos líticos involucrados, con el objeto de controlar ciertos aspectos estructurales y organizativos. De este modo, se podrán generar conocimientos acerca de las estrategias de aprovisionamiento implementadas, el estado del bien intercambiado (materia prima no modificada, núcleos y/o formas base, productos terminados) y la intensidad del tráfico a través del tiempo.

En este sentido, estudios tecnológicos efectuados sobre el conjunto artefactual del sitio Casa Chávez Montículos puede constituir una primera aproximación a estas cuestiones. Este sitio se localiza en el fondo de cuenca, a $1.7-2 \mathrm{~km}$ al sur de la localidad de Antofagasta de la Sierra, en el margen izquierdo del río Antofagasta/Punilla y adyacente a la zona Tolar-Campo. Se trata de un conjunto de 10 estructuras monticulares, de dimensiones variables, dispuestas en dos grupos alrededor de un espacio deprimido central. Los registros de excavación provienen fundamentalmente de los Montículos 1 y 4, siendo el Montículo 1 el que presenta la secuencia más larga. Los fechados radiocarbónicos disponibles permiten situar las ocupaciones del sitio entre 2120 y 1530 AP (Tabla 2). Si se contemplan estas dataciones y dos sigmas es posible hablar de un lapso probable de ocupación de más de 850 años, dado que el fechado más temprano en el Nivel VIII se encuentra por encima de la base de la ocupación (Nivel $\mathrm{X})$. En líneas generales, la existencia de evidencias de agricultura, manejo selectivo de camélidos y tecnología cerámica desarrollada, sumadas a una larga secuencia de ocupación, advierten acerca de la existencia de una comunidad agropastoril con alto grado de sedentarismo en este sitio (Olivera 1992, 1998).

Una manera de abordar las características estructurales y organizativas en los conjuntos artefactuales es a través de la delineación de trayectorias de producción (Koldehoff 1987). Dichas trayectorias, planteadas como modelos particulares, apuntan a clarificar la relación entre la disponibilidad de recursos líticos, las secuencias de producción y la distribución espacial de las actividades productivas. En estudios previos efectuados sobre muestras artefactuales fueron desarro-

\begin{tabular}{|lccl|}
\hline $\begin{array}{l}\text { Sitio } \\
\text { Casa Chávez } \\
\text { Montículos }\end{array}$ & Nivel & $\begin{array}{c}\text { Edad C } \\
\text { Años AP }\end{array}$ & $\begin{array}{c}\text { Laboratorio } \\
\text { y muestra }\end{array}$ \\
\hline Montículo 1 & III & $1670 \pm 60$ & Beta -27199 \\
Montículo 1 & IV & $1660 \pm 60$ & Latyr -251 \\
Montículo 1 & Vc & $1530 \pm 70$ & Beta -27201 \\
Montículo 1 & VI & $1740 \pm 60$ & Beta -27202 \\
Montículo 1 & VII & $1930 \pm 70$ & Beta -27200 \\
Montículo 1 & VIII & $2120 \pm 60$ & Latyr -299 \\
\hline
\end{tabular}

Tabla 2. Fechados radiocarbónicos.

lladas cuatro trayectorias correspondientes a las principales materias primas, entre las cuales se encontraba la obsidiana de Ona (Escola 1999, 2000). En esta oportunidad, y para afinar más los resultados ya obtenidos, se definen las trayectorias de producción para las obsidianas de Ona y Cueros de Purulla. Para esta tarea, por un lado, se tomaron en consideración las observaciones de campo realizadas en la fuente Ona y, por otro, se utilizó la información resultante del análisis del total de los artefactos de obsidiana de ambas fuentes recuperados en CChM1 ( $\mathrm{n}=2870)$. En este sentido, siguiendo la clasificación de Aschero (1975 Ms, 1983 Ms), se privilegiaron los siguientes atributos: a) para instrumentos y núcleos: materia prima (variedad de obsidiana en este caso), grupo tipológico, tamaño relativo, designación morfológica (núcleos), situación de los lascados, forma base; b) para desechos de talla: estado de desecho, tamaño relativo, tipo de lasca, reserva de corteza.

Antes de pasar a las trayectorias considero importante aclarar la frecuencia de aparición de las obsidianas respecto de las restantes materias primas identificadas $(n=12)$ pero examinando ahora el total artefactual del sitio (531 instrumentos y núcleos y 10443 desechos de talla). En el caso de Ona, la obsidiana sobresale con un porcentaje del $25.0 \%$, mientras que la de Cueros de Purulla muestra una frecuencia de $1.0 \%(\mathrm{n}=10974)$.

La trayectoria productiva de la obsidiana de Ona se inicia en la fuente de aprovisionamiento. Depósitos primarios y secundarios detectados muestran a la obsidiana en forma de nódulos, de variados tamaños y coloraciones. En términos generales, las observaciones y análisis efectuados en el área de aprovisionamiento sugieren la realización de las siguientes actividades productivas: 
- testeo y selección de nódulos viables para su procesamiento

- descortezamiento primario y preparación de núcleos para su transporte

- extracción de formas base o lascas de tamaños grandes y muy grandes factibles de ser transportadas a otras localizaciones.

Ya en la microrregión de Antofagasta de la Sierra el conjunto lítico de la base residencial del fondo de cuenca (CChM1) registra el ingreso de la obsidiana bajo la forma de núcleos preparados y, posiblemente, de formas base para instrumentos. Estos núcleos, orientados esencialmente a la obtención de lascas pequeñas y mediano pequeñas, son objeto de reactivaciones (presencia de lascas de reactivación de núcleos) y de intensas reducciones. En este sentido, se puede advertir que, como último recurso para extraer formas base, se impone la utilización de la talla bipolar a fin de extremar la utilidad del recurso (Flegenheimer et al. 1995; Hayden 1980) En esta misma dirección, algunos núcleos, llegados al punto de agotamiento parcial o total, llegan a convertirse, en sí mismos, en formas base de instrumentos de manufactura simple, con retoque marginal unifacial (p.e., muescas y artefactos mediano pequeños/muy pequeños, retoque en bisel oblicuo). Cabe señalar que respecto del total de instrumentos y núcleos de obsidiana Ona $(n=113)$ (Tabla 3$)$, se ha registrado una frecuencia de $13.3 \%$ (15 piezas) de nú- cleos y fragmentos de núcleos, pequeños y mediano pequeños, agotados y parcialmente agotados, de los cuales el $60.0 \%$ (9 piezas) son bipolares. Asimismo, en cuanto a la posible entrada de formas base a CChM1, se evalúa en tal sentido la presencia de lascas de mayor tamaño (1.2\% del total de desechos de obsidiana Ona, $\mathrm{n}=2648$ ) que el rango instrumental (pequeño y mediano pequeño).

La búsqueda de soportes adecuados para la manufactura de proyectiles parece ser el eje direccional del procesamiento de esta materia prima. En este sentido, se puede apreciar que una gran mayoría de las lascas producidas se utiliza fundamentalmente para la manufactura de un instrumento formatizado y regularizado por medio de retoques bifaciales extendidos o parcialmente extendidos. Me refiero específicamente a puntas de proyectil pedunculadas y apedunculadas de base escotada. Obsérvese en la Tabla 3 que, dentro del total instrumental de la obsidiana Ona ( $\mathrm{n}=98$ sin núcleos) representado por 14 grupos tipológicos, las puntas de proyectil (enteras, fracturadas y preformas) constituyen el $38.77 \%$. Estas puntas de proyectil son, en su gran mayoría, pequeñas, mientras que los grupos tipológicos restantes son pequeños y mediano pequeños predominantemente.

Sin embargo, una vez agotadas las posibilidades tecnológicas de mantener esta orientación, se ad-

\begin{tabular}{|c|c|c|c|c|c|c|}
\hline \multirow[t]{2}{*}{ Grupos tipológicos } & \multicolumn{2}{|c|}{ Ona } & \multicolumn{2}{|c|}{ Cueros de Purulla } & \multicolumn{2}{|c|}{ Totales } \\
\hline & $\overline{\mathbf{N}}$ & $\%$ & $\mathbf{N}$ & $\%$ & $\overline{\mathbf{N}}$ & $\%$ \\
\hline Bifaces & & & 1 & 14.3 & 1 & 0.8 \\
\hline Raspadores & 4 & 3.5 & & & 4 & 3.3 \\
\hline Artef. bisel asimétrico RUM & 6 & 5.3 & & & 6 & 5.0 \\
\hline Artef. med. peq./muy peq. RBO & 8 & 7.1 & & & 8 & 6.8 \\
\hline Cuchillos de filo retocado & & & 1 & 14.3 & 1 & 0.8 \\
\hline Cortantes & 2 & 1.8 & & & 2 & 1.7 \\
\hline Muescas retocadas y de lascado simple & 4 & 3.5 & & & 4 & 3.3 \\
\hline Denticulados & 2 & 1.8 & & & 2 & 1.7 \\
\hline Puntas entre muescas & 1 & 0.9 & & & 1 & 0.8 \\
\hline Artefactos burilantes & 3 & 2.6 & & & 3 & 2.5 \\
\hline Puntas de proyectil y preformas & 38 & 33.6 & 1 & 14.3 & 39 & 32.5 \\
\hline Filos naturales con rastros complementarios & 7 & 6.2 & & & 7 & 5.8 \\
\hline Artefactos de formatización sumaria & 2 & 1.8 & & & 2 & 1.7 \\
\hline Frag. no dif. de artef. formatizados & 21 & 18.6 & 3 & 42.8 & 24 & 20.0 \\
\hline Núcleos & 15 & 13.3 & 1 & 14.3 & 16 & 13.3 \\
\hline Totales & 113 & 100 & 7 & 100 & 120 & 100 \\
\hline
\end{tabular}

Tabla 3. Distribución de grupos tipológicos por variedad de obsidiana (sitio CChM1). Referencias: Artef. bisel asimétrico RUM: Artefacto bisel asimétrico con microrretoque ultramarginal; Artef. med. peq./muy peq. RBO: Artefacto mediano pequeño/muy pequeño con retoque en bisel oblicuo; Frag. no dif. de artef. formatizados: Fragmentos no diferenciados de artefactos formatizados. 
vierte la instrumentación de otras alternativas en función de la maximización del recurso. Lejos de descartar aquellos subproductos poco adecuados como soportes de los proyectiles, éstos se utilizaron para la manufactura de instrumentos simples en el marco de un comportamiento económico. De este modo, en menor medida, lascas externas e internas han sido modificadas para producir algunos instrumentos unifaciales confeccionados con procedimientos de retoque marginal y/o ultramarginal (artefactos mediano pequeños/muy pequeños, retoque en bisel oblicuo, artefactos con filos en bisel asimétrico y microrretoque ultramarginal, raspadores, artefactos burilantes, muescas, denticulados, cortantes, puntas entre muescas).

Finalmente, cabe destacar que todas estas actividades productivas han generado y dejado en contexto un total de 2648 desechos de talla de obsidiana Ona comprendiendo el $25.3 \%$ del total de desechos del conjunto lítico general. Al respecto, tal como se aprecia en la Tabla 4, predominan las lascas internas, a las que siguen en orden decreciente de abundancia, los productos bipolares y las lascas externas con tamaños que van desde los muy pequeños $(48.1 \%)$, pasando por los pequeños $(43.2 \%)$, mediano pequeños $(7.5 \%)$, mediano grandes $(1.0 \%)$ y grandes $(0.2 \%)$.

Por su parte, la trayectoria productiva de la obsidiana de Cueros de Purulla se iniciaría también en la fuente de aprovisionamiento, aunque no tenemos datos de las actividades productivas realizadas allí hasta el momento. Lo cierto es que en CChM1 hay evidencia de la entrada de núcleos (un núcleo discoidal irregular de tamaño grande) $\mathrm{y}$, en principio, se podría decir que hay evidencias de la manufactura in situ de instrumental. Al respecto, es posible que estos núcleos estuvieran en parte descortezados ya que no hay evidencia de instrumentos confeccionados sobre lascas externas, pero sí se han consignado lascas externas de tamaño pequeño. El registro instrumental $(n=6$, sin núcleos) comprende una punta de proyectil pedunculada, un bifaz ${ }^{2}$, un fragmento de cuchillo $\mathrm{y}$ tres instrumentos fracturados no diferenciados,

2 Considero importante señalar que el bifaz recuperado no ha sido elaborado por procedimientos de reducción o adelgazamiento bifacial. En este caso puede observarse simplemente en las caras de la pieza la presencia de retoques parcialmente extendidos con negativos profundos y restos de corteza. cuyos tamaños responden a piezas mediano pequeñas o algo mayores, en el caso de los fracturados (Tabla 3). Este reducido conjunto instrumental no parecería evidenciar una utilización selectiva de la obsidiana Cueros de Purulla para la manufactura de instrumentos particulares, tal el caso de la obsidiana Ona y las puntas de proyectil.

En cuanto a los subproductos de talla, se consigna la presencia de un total de 102 desechos. Al respecto, la Tabla 4 muestra el predominio de las lascas internas, seguidas con frecuencias menores por los productos bipolares y las lascas externas. Sus tamaños van desde los pequeños (46.1\%), los muy pequeños $(44.1 \%$ ) hasta los mediano pequeños $(9.8 \%)$. Es interesante destacar la presencia de los productos bipolares ya que podría ser indicativa de actividades orientadas a la preservación o maximización de esta materia prima.

Ahora bien, dentro del registro rescatado en CChM1 existen ciertos elementos que han permitido plantear la existencia de dos componentes ergológicos diferentes. La separación de ambos componentes coincidiría con un episodio de desocupación temporaria detectado entre los niveles V y VI de la estratigrafía. El componente inferior (Niveles VI a XI) estaría evidenciando ciertas similitudes contextuales con ocupaciones de las quebradas altas del Noroeste Argentino (valle Calchaquí y quebrada del Toro) y el norte de Chile (región del Loa). Por otra parte, el componente superior (Niveles I a V) mostraría un significativo aumento en la intensidad de las relaciones con los valles mesotermales del Noroeste Argentino, especialmente Hualfín y Abaucán (Olivera 1991, 1992).

Esta separación, tomando en consideración los fechados disponibles (Tabla 2), coincide con dos de los segmentos cronológicos analizados en los estudios de procedencia (2200-1800 AP y $1800-$ 1100 AP), de modo tal que si se examinan los datos tecnológicos registrados para ambas obsidianas por componente se podrá hacer una evaluación más profunda acerca de la intensidad de la circulación de estos bienes y sus posibles implicancias socioeconómicas.

En principio, si se toma en consideración el total de la producción de ambas variedades de obsidianas $(\mathrm{n}=2870)$ y se compara la frecuencia de aparición de cada una de ellas por componente tenemos los siguientes resultados: en el compo- 


\begin{tabular}{|c|c|c|c|c|c|c|}
\hline \multirow[t]{2}{*}{ Desechos de talla } & \multicolumn{2}{|c|}{ Ona } & \multicolumn{2}{|c|}{ Cueros de Purulla } & \multicolumn{2}{|c|}{ Totales } \\
\hline & $\overline{\mathbf{N}}$ & $\overline{\%}$ & $\mathbf{N}$ & $\%$ & $\overline{\mathbf{N}}$ & $\overline{\%}$ \\
\hline Lascas externas & 26 & 1.0 & 3 & 2.9 & 29 & 1.1 \\
\hline Lascas internas & 2373 & 89.6 & 94 & 92.2 & 2467 & 89.7 \\
\hline Productos bipolares & 122 & 4.6 & 3 & 2.9 & 125 & 4.5 \\
\hline Desechos indiferenciados & 127 & 4.8 & 2 & 2.0 & 129 & 4.7 \\
\hline Totales & 2648 & 100 & 102 & 100 & 2750 & 100 \\
\hline
\end{tabular}

Tabla 4. Desechos de talla por variedad de obsidiana (sitio CChM1).

nente inferior, es decir, en el segmento temporal de 2200-1800 AP, la obsidiana de Ona aparece con un $95.2 \%$ mientras que la de Cueros de Purulla con $4.8 \%$. Por su parte, en el componente superior (1800-1100 AP) la intensidad de uso de ambas obsidianas varía diferencialmente, registrándose un leve aumento para Ona con $97.6 \%$ y una leve reducción para Cueros de Purulla con $2.4 \%$. Por otro lado, es interesante advertir que la obsidiana de Ona aparece en todos los niveles estratigráficos, observándose en el componente superior tanto instrumentos como desechos, mientras que en el componente inferior los instrumentos se presentan hasta el nivel IX y los desechos en todos sus niveles. A su vez, la obsidiana de Cueros de Purulla tiene continuidad en la secuencia de ocupación sólo en el registro de desechos hasta el nivel IX mientras que los instrumentos han sido registrados en el nivel IV (componente superior) y en los niveles VII, VIII y IX (componente inferior).

Teniendo presente lo anterior, ¿cuál ha sido el manejo de estas materias primas en los dos componentes? En el caso de la obsidiana de Ona, se observa en el componente inferior una menor cantidad de núcleos ( $\mathrm{n}=5$, la mitad de piezas de las que aparecen en el componente superior) pero presentando mayor variabilidad morfológica. En este sentido, se registran dos núcleos bipolares, uno piramidal irregular, uno prismático bidireccional y un fragmento no determinado. Cabe destacar que el componente superior muestra la presencia de 10 núcleos enteros y fragmentados, de los cuales ocho son bipolares, evidencia a la que se suma la existencia de un mayor porcentaje de productos bipolares respecto del componente anterior $(6.6 \%$ de productos bipolares en el componente superior versus $3.2 \%$ para el inferior) (Tablas 5, 6, 7 y 8). La evidencia de ambos componentes, may or variabilidad morfológica versus alta frecuencia de núcleos bipolares, podría estar indicando distintas estrategias técnicas para lograr un aprovechamiento más económico del material entrante

En lo que respecta a los instrumentos (Tabla 5 y 7), se puede decir que ambos componentes involucran un variado grupo de clases instrumentales. Sin embargo, es interesante destacar que, en el componente inferior $(n=42$ sin núcleos), las puntas de proyectil registran el $42.8 \%$, concentrándose más del $80 \%$ de las mismas en el nivel VI, nivel que ostenta la mayor frecuencia de aparición de desechos de talla. Este mayor porcentaje de puntas podría estar en relación con la predominante presencia mensurable de desechos de tamaño muy pequeño en este componente respecto del anterior $(59.3 \% ; \mathrm{n}=1571$ versus $31.7 \%$; $\mathrm{n}=1077$ ). Cabe señalar que la manufactura de estos proyectiles, de tamaño pequeño y mediano pequeño, por técnica de presión genera subproductos de talla como el mencionado anteriormente. Asimismo, no hay que olvidar que en estos contextos tecnológicamente expeditivos las puntas de proyectil son los instrumentos que mayor inversión de trabajo conllevan respecto de los restantes (Escola 2000). Esto se hace extensivo también para el componente superior ( $\mathrm{n}=56$, sin núcleos), en donde las puntas de proyectil, una vez más, constituyen el grupo tipológico más representado con un $35.7 \%$.

En el caso de la obsidiana de Cueros de Purulla, se observa en el componente inferior (que registra la mayor disponibilidad del recurso) una apreciable variabilidad artefactual tanto en el registro instrumental como en el de desechos a los que se suma la presencia del único núcleo de lascas recuperado (Tablas 5 y 6). Si bien entre los instrumentos se registra una punta de proyectil pedunculada no hay evidencia clara de que esta materia 


\begin{tabular}{|c|c|c|c|c|c|c|}
\hline \multirow[t]{2}{*}{ Grupos tipológicos } & \multicolumn{2}{|c|}{ Ona } & \multicolumn{2}{|c|}{ Cueros de Purulla } & \multicolumn{2}{|c|}{ Totales } \\
\hline & $\overline{\mathbf{N}}$ & $\overline{\%}$ & $\frac{N}{N}$ & $\%$ & $\overline{\mathbf{N}}$ & $\overline{\%}$ \\
\hline Bifaces & & & 1 & 20.0 & 1 & 1.9 \\
\hline Raspadores & 2 & 4.3 & & & 2 & 3.9 \\
\hline Artef. bisel asimétrico RUM & 2 & 4.3 & & & 2 & 3.9 \\
\hline Artef. med. peq./muy peq. RBO & 4 & 8.5 & & & 4 & 7.6 \\
\hline Cuchillos de filo retocado & & & 1 & 20.0 & 1 & 1.9 \\
\hline Cortantes & 2 & 4.3 & & & 2 & 3.9 \\
\hline Muescas retocadas y de lascado simple & 1 & 2.1 & & & 1 & 1.9 \\
\hline Denticulados & 1 & 2.1 & & & 1 & 1.9 \\
\hline Artefactos burilantes & 2 & 4.3 & & & 2 & 3.9 \\
\hline Puntas de proyectil y preformas & 18 & 38.2 & 1 & 20.0 & 19 & 36.5 \\
\hline Filos naturales con rastros complementarios & 2 & 4.3 & & & 2 & 3.9 \\
\hline Frag. no dif. de artef. formatizados & 8 & 17.0 & 1 & 20.0 & 9 & 17.3 \\
\hline Núcleos & 5 & 10.6 & 1 & 20.0 & 6 & 11.5 \\
\hline Totales & 47 & 100 & 5 & 100 & 52 & 100 \\
\hline
\end{tabular}

Tabla 5. Grupos tipológicos por variedad de obsidiana (Componente Inferior de CChM1). Referencias: Artef. bisel asimétrico RUM: Artefacto bisel asimétrico con microrretoque ultramarginal; Artef. med. peq./muy peq. RBO: Artefacto mediano pequeño/muy pequeño con retoque en bisel oblicuo; Frag. no dif. de artef. formatizados: Fragmentos no diferenciados de artefactos formatizados.

\begin{tabular}{|c|c|c|c|c|c|c|}
\hline \multirow[t]{2}{*}{ Desechos de talla } & \multicolumn{2}{|c|}{ Ona } & \multicolumn{2}{|c|}{ Cueros de Purulla } & \multicolumn{2}{|c|}{ Totales } \\
\hline & $\mathbf{N}$ & $\%$ & $\mathbf{N}$ & $\%$ & $\mathbf{N}$ & $\%$ \\
\hline Lascas externas & 9 & 0.6 & 1 & 1.3 & 10 & 0.6 \\
\hline Lascas internas & 1452 & 92.4 & 71 & 93.5 & 1523 & 92.5 \\
\hline Productos bipolares & 51 & 3.2 & 3 & 3.9 & 54 & 3.3 \\
\hline Desechos indiferenciados & 59 & 3.8 & 1 & 1.3 & 60 & 3.6 \\
\hline Totales & 1571 & 100 & 76 & 100 & 1647 & 100 \\
\hline
\end{tabular}

Tabla 6. Desechos de talla por variedad de obsidiana (Componente Inferior de CChM1).

\begin{tabular}{|c|c|c|c|c|c|c|}
\hline \multirow{2}{*}{ Grupos tipológicos } & \multicolumn{2}{|c|}{ Ona } & \multicolumn{2}{|c|}{ Cueros de Purulla } & \multicolumn{2}{|c|}{ Totales } \\
\hline & $\overline{\mathbf{N}}$ & $\%$ & $\mathbf{N}$ & $\%$ & $\overline{\mathbf{N}}$ & $\%$ \\
\hline Raspadores & 2 & 3.0 & & & 2 & 2.9 \\
\hline Artef. bisel asimétrico RUM & 4 & 6.1 & & & 4 & 5.9 \\
\hline Artef. med. peq./muy peq. RBO & 4 & 6.1 & & & 4 & 5.9 \\
\hline Muescas retocadas y de lascado simple & 3 & 4.5 & & & 3 & 4.4 \\
\hline Denticulados & 1 & 1.5 & & & 1 & 1.5 \\
\hline Puntas entre muescas & 1 & 1.5 & & & 1 & 1.5 \\
\hline Artefactos burilantes & 1 & 1.5 & & & 1 & 1.5 \\
\hline Puntas de proyectil y preformas & 20 & 30.3 & & & 20 & 29.4 \\
\hline Filos naturales con rastros complementarios & 5 & 7.6 & & & 5 & 7.4 \\
\hline Artefactos de formatización sumaria & 2 & 3.0 & & & 2 & 2.9 \\
\hline Frag. no dif. de artef. formatizados & 13 & 19.7 & 2 & 100 & 15 & 22.1 \\
\hline Núcleos & 10 & 15.2 & & & 10 & 14.7 \\
\hline Totales & 66 & 100 & 2 & 100 & 68 & 100 \\
\hline
\end{tabular}

Tabla 7. Grupos tipológicos por variedad de obsidiana (Componente Superior de CChM1). Referencias: artef. bisel asimétrico RUM: Artefacto bisel asimétrico con microrretoque ultramarginal; Artef. med. peq./muy peq. RBO: Artefacto mediano pequeño/muy pequeño con retoque en bisel oblicuo; Frag. no dif. de artef. formatizados: Fragmentos no diferenciados de artefactos formatizados. 


\begin{tabular}{|c|c|c|c|c|c|c|}
\hline \multirow[t]{2}{*}{ Desechos de talla } & \multicolumn{2}{|c|}{ Ona } & \multicolumn{2}{|c|}{ Cueros de Purulla } & \multicolumn{2}{|c|}{ Totales } \\
\hline & $\overline{\mathbf{N}}$ & $\%$ & $\mathbf{N}$ & $\%$ & $\overline{\mathbf{N}}$ & $\%$ \\
\hline Lascas externas & 17 & 1.6 & 2 & 7.7 & 19 & 1.7 \\
\hline Lascas internas & 921 & 85.5 & 23 & 88.5 & 944 & 85.6 \\
\hline Productos bipolares & 71 & 6.6 & & & 71 & 6.4 \\
\hline Desechos indiferenciados & 68 & 6.3 & 1 & 3.8 & 69 & 6.3 \\
\hline Totales & 1077 & 100 & 26 & 100 & 1103 & 100 \\
\hline
\end{tabular}

Tabla 8. Desechos de talla por variedad de obsidiana (Componente Superior de CChM1).

prima se utilizara selectivamente para la confección de estas piezas tal como sucede con la obsidiana de Ona. Sin embargo, es de destacar que el proyectil mencionado, registrado en el nivel VIII (Tabla 2), no responde al diseño recurrente en la secuencia de CChM1, sino a un diseño romboidal, espeso, que también aparece en el sitio Las Cuevas (Quebrada del Toro), con cronología similar (Escola 1991).

Considero importante señalar que la mayor presencia instrumental en este componente inferior se ve acompañada no sólo por una superior frecuencia de desechos de talla, sino también por una elevada proporción de desechos de tamaños muy pequeños $^{3}$ en comparación con el componente superior $(51.3 \%$; $\mathrm{n}=76$ versus $23.1 \%$; $\mathrm{n}=26)$. Asimismo, entre los desechos del componente inferior se registran algunas evidencias de trabajo bipolar (Tabla 6). Por su parte, el componente superior sólo registra la presencia de dos fragmentos no diferenciados de artefactos formatizados y 26 desechos de talla predominantemente pequeños (69.2\%), seguidos por los muy pequeños $(23.1 \%)$ y mediano pequeños $(7.7 \%$ ) (Tablas 7 y 8 ).

\section{Conclusiones}

La información obtenida a través de la delineación de las trayectorias productivas estaría indicando la circulación paralela de las obsidianas de Ona y Cueros de Purulla, observándose una intensidad diferencial que se inclina notoriamente en favor de la obsidiana de Ona $(96.2 \%$ para Ona versus $3.8 \%$ para Cueros de Purulla, siendo $\mathrm{n}=2870$ ). Asimismo, es posible sostener que la

3 Los desechos de talla restantes muestran en orden decreciente tamaños pequeños $(38.2 \%)$ y mediano pequeños $(10.5 \%)$ obsidiana en ambos casos habría circulado no como productos terminados sino como núcleos $(\mathrm{y}$ posiblemente como formas base en el caso de Ona), registrándose evidencias de manufactura local de instrumentos. Esto significa que el registro artefactual analizado no responde a la exclusiva presencia de instrumentos terminados, sino que comprende el desarrollo de casi todas las instancias de reducción y descarte.

En el caso de la obsidiana de Ona, se puede decir que ha sido utilizada selectivamente para la confección de instrumentos complejos y estandarizados como las puntas de proyectil y, a su vez, aprovechada de manera económica para la manufactura de variados instrumentos simples. Por su parte, el reducido registro artefactual de la obsidiana de Cueros de Purulla no parece presentar tal selectividad. Sin embargo, no puede dejar de advertirse, nuevamente, la presencia conjunta de una punta de proyectil de diseño particular y algunos instrumentos simples.

Con respecto a la variación temporal en el uso de ambas variedades de obsidiana, considero importante destacar que la dominancia de la obsidiana Ona y el aporte minoritario de la obsidiana Cueros de Purulla se mantiene constante a lo largo de los dos componentes estudiados. Asimismo, tomando en consideración los dos segmentos cronológicos analizados, es posible advertir que la obsidiana de Ona muestra un flujo más intenso en el lapso 1800-1100 AP, mientras que, por el contrario, la de Cueros de Purulla evidencia cierto incremento en su consumo durante 2200-1800 AP. En este sentido, resulta interesante señalar que, siguiendo la información de procedencia disponible, en el segmento temporal más antiguo ambas variedades de obsidiana se muestran tanto en la puna como en los valles (valle del Cajón) (Tabla 1 y Figura 1). Luego, en momentos posteriores (18001100 AP), la distribución parece modificarse. La 
obsidiana Ona circula en el sector puneño al igual que en los valles, llegando a su máxima dispersión en las tierras bajas del este como lo muestra el sitio Ampascachi. En contraste, la obsidiana Cueros de Purulla parece restringir su distribución respecto del segmento anterior localizándose exclusivamente en la puna (ver Tabla 1 y Figura 1).

Por otra parte, evaluando otro aspecto del consumo de estas obsidianas en el tiempo, se debe destacar que la obsidiana de Ona presenta una estructura tipológica similar y una misma orientación tecnológica a lo largo de los dos componentes analizados. Por el contrario, la obsidiana Cueros de Purulla muestra una variedad artefactual en el componente inferior (o segmento temporal más antiguo) que no se mantiene en el componente superior.

Ahora, las observaciones realizadas acerca del estado en que habrían circulado las obsidianas y las características de su consumo, ¿podrían hacerse extensivas a otros contextos agropastoriles del Noroeste Argentino? La respuesta es aún incierta dada la escasez de estudios tecnológicos sobre registros líticos agroalfareros. Sin embargo, los datos obtenidos por Lazzari (1997: 23-28) para la obsidiana de Ona en la falda occidental del Aconquija (Catamarca), distante 250-260 km de la fuente, podrían marcar una tendencia positiva a este respecto. La autora sugiere que los materiales de Ona habrían viajado desde su fuente en forma de núcleos preparados, los que habrían sido modificados en la falda dadas las evidencias de manufactura local de instrumentos. En este sentido, en lo que respecta a la variedad de productos finales, señala específicamente que habría para la obsidiana de Ona un rango muy pequeño y específico de usos sobresaliendo una vez más la presencia de puntas de proyectil.

Sin lugar a dudas, para profundizar la tendencia marcada en cuanto a este bien de intercambio en circulación, resulta imprescindible incrementar los estudios líticos en los distintos sectores del Noroeste Argentino que registren presencia de ambas obsidianas. Más aún, estos estudios no deberán perder de vista el hecho de que la tecnología lítica, al igual que otras tradiciones de confección y uso, es modelada no sólo por las demandas prácticas sino también por las relaciones sociales y políticas (Edmonds 1995). En este sentido, resulta interesante advertir que, como materiales aptos para la confección de instrumentos, la obsidiana Cueros de Purulla es de calidad muy superior a la de Ona. Sin embargo, es esta última la que domina en cuanto a rangos de distribución e intensidad de consumo. Por otro parte, en el caso analizado de Casa Chávez Montículos, factores tales como la distancia y las condiciones de accesibilidad no brindan explicación suficiente para las diferencias observadas en el consumo de ambas obsidianas. Ambas fuentes se encuentran a distancias similares, siendo Cueros de Purulla la más cercana, y el acceso a las mismas no presenta diferencias relevantes. Entonces, ¿existirá la posibilidad de que el valor social asignado a estas obsidianas sea diferente? Este interrogante deberá guiar las investigaciones futuras y constituirse en un reto a desentrañar dentro de la dimensión del intercambio.

Agradecimientos A Andrea Seelenfreund y Teresa Civalero por haberme dado la posibilidad de participar del evento que dió origen a este volumen. A Jorge Reales por brindarme su colaboración en la parte gráfica. A Gabriela Guráieb por ocuparse de un envío que fue crucial para terminar este trabajo. Al Consejo Nacional de Investigaciones Científicas y Técnicas (CONICET) y a la National Geographic Foundation sin cuyo apoyo económico este trabajo no podría haberse realizado. A la comunidad del Departamento de Antofagasta de la Sierra por su colaboración y hospitalidad.

\section{REFERENCIAS CITADAS}

ASCHERO, C., 1975 Ms. Ensayo para una clasificación morfológica de artefactos líticos aplicada a estudios tipológicos comparativos. Informe presentado al Consejo Nacional de Investigaciones Científicas y Técnicas (CONICET), Buenos Aires.
1983 Ms. Ensayo para una clasificación morfológica de artefactos líticos. Apéndices A y B. Cátedra de Ergología y Tecnología, Facultad de Filosofía y Letras, Universidad de Buenos Aires, Buenos Aires. 
ASCHERO, C., P. ESCOLA, S. HOCSMAN y J. MARTINEZ, 2002. Recursos líticos en escala microrregional. Antofag asta de la Sierra. 1983-2001. Arqueología 12. En prensa.

COBEAN, R. H., J. R. VOGT, M. D. GLASCOCK y T. L. STOCKER, 1991. High precision trace element characterization of major mesoamerican obsidian sources and further analysis of artifacts from San Lorenzo Tecnochtitlán, Mexico. Latin American Antiquity (2): 69-91.

EDMONDS, M., 1995. Stone tools and society. B. T. Bratsford Ltd., Londres.

ESCOLA, P., 1991. Puntas de proyectil de contextos formativos: Acercamiento tecno-tipológico a través de cuatro casos de análisis. Actas del XI Congreso Nacional de Arqueología Chilena (1988), T. II: 175-184. Santiago.

_ 1999. La variable tecnológica en contextos agropastoriles. Humanitas, número extraordinario, Año XXII, pp. 49-76.

-2000. Tecnología lítica y contextos agropastoriles tempranos. Tesis para optar al grado de Doctor en Filosofía y Letras. Facultad de Filosofía y Letras. Universidad de Buenos Aires, Buenos Aires.

FERNANDEZ, M., 1992. The social organization of production in community-based agropastoralism in the Andes. En Plants, animals, and people. Agropastoral systems research, C. McCorkle (Ed.), pp. 99-110. Westview Press, Boulder.

FLEGENHEIMER, N., C. BAYON y M. I. GONZALEZ DE BONAVERI, 1995. Técnica simple, comportamientos complejos: La talla bipolar en la arqueología bonaerense. Relaciones de la Sociedad Argentina de Antropología XX: 81-110.

GLASCOCK, M. D., 2002. Introduction: Geochemical evidence for long-distance exchange. En Geochemical evidence for long-distance exchange, M. D. Glascock (Ed.), pp. 1-11. Bergin and Garvey, Wesport, Connecticut.

GONZALEZ, O. E., 1992. Geología de la puna austral entre $\operatorname{los} 25^{\circ} 15^{\prime}$ a $26^{\circ} 30^{\prime}$ de Lat. Sur y $\operatorname{los} 66^{\circ} 25^{\prime}$ a $68^{\circ} 00^{\prime}$ de Long. Oeste, provincias de Catamarca y Salta, Argentina. Acta Geológica Lilloana XVII (2): 63-88.

HAYDEN, B., 1980. Confusion in the bipolar world: Bashed pebbles and splintered pieces. Lithic Technology 9 (1): $2-7$.

HUGHES, R. E., 1998. On reliability, validity, and scale in obsidian sourcing research. En Unit issues in archaeology, A. N. Ramenofsky y A. Steffan (Eds.), pp. 103-104. The University of Utah Press, Salt Lake City.

HURTADO DE MENDOZA, L., 1986. La obsidiana de El Chayal y su redistribución en Kaminaljuyú (Guatemala), 0-200 DC. Revista Mesoamericana 12: 395-415.
HURTADO DE MENDOZA, L. y W. A. JESTER, 1978. Obsidian sources in Guatemala: A regional approach. American Antiquity 47 (1): 159-172.

KOLDEHOFF, B., 1987. The Cahokia flake tool industry: Socioeconomic implications for Late Prehistory in the central Mississippi valley. En The organization of core technology. J. K. Johnson y C. A. Morrow (Eds.), pp. 151-185. Westview Press, Boulder.

LAZZARI, M., 1997. La economía más allá de la subsistencia: Intercambio y producción en el Aconquija. Arqueología 7: 9-50.

OLIVERA, D., 1991. El Formativo en Antofagasta de la Sierra (Puna Meridional argentina): Análisis de sus posibles relaciones con contextos arqueológicos agroalfareros tempranos del Noroeste Argentino y norte de Chile. Actas del XI Congreso Nacional de Arqueología Chilena (1988), T. II: 61-78. Santiago.

-1992. Tecnología y estrategias de adaptación en el Formativo (Agroalfarero) Temprano de la Puna Meridional argentina. Un caso de estudio: Antofagasta de la Sierra (provincia de Catamarca, Argentina). Tesis para optar al grado de Doctor en Ciencias Naturales. Facultad de Ciencias Naturales, Universidad Nacional de La Plata, La Plata.

1998. Cazadores y pastores tempranos de la puna arg entina. En Past and present in Andean prehistory and early history, S. Ahlgren, A. Muñoz, S. Sjödin y P. Stenborg (Eds.), pp. 153-180. Etnologiska Studier 42, Göteborg.

O'SHEA, J. M., 1989. The role of wild resources in smallscale agrocultural systems: Tales from the lakes and the plains. En Bad year economics: Cultural responses to risk and uncertainty, P. Halstead y J. O'Shea (Eds.), pp. 57-67. Cambridge University Press, Cambridge.

ROWLEY-CONWY, P. y M. ZVELEBIL, 1989. Saving it for later: Storage by prehistoric hunter-gatherers in Europe. En Bad year economics: Cultural responses to risk and uncertainty, P. Halstead y J. O'Shea (Eds.), pp. 40-56. Cambridge University Press, Cambridge.

SANTORO, C. y L. NUÑEZ, 1987. Hunters of the Dry Puna and the Salt Puna in Northern Chile. Andean Past 1: 57109.

SCARRE, C., 1993. Introduction. En Trade and exchange in prehistoric Europe, C. Scarre y F. Healy (Eds.), pp. 1-4. Oxbow Monograph 33. Oxbow Books, Oxford.

SIDRYS, R. V., J. ANDERSON y D. MARCUCCI, 1976. Obsidian sources in the Maya area. Journal of New World Archaeology 1 (5): 1-13.

VIRAMONTE, J., H. DESTEFANI, A. APARICIO, R. ALONSO, J. MARCUZZI, E. CINCIEONI e I. PETRINOVIC, 1988. Caracterización y beneficios de perlitas del N.O.A. Actas III Congreso Nacional de Geología Económica, pp. 549-566. Olavarría. 
YACOBACCIO, H., P. ESCOLA, M. LAZZARI y F. X. PEREYRA, 2002. Long-distance obsidian traffic in Northwestern Argentina. En Geochemical evidence for long-distance exchange, M. D. Glascock (Ed.), pp. 167203. Bergin and Garvey, Wesport, Connecticut.
YACOBACCIO, H., P. ESCOLA, F. X. PEREYRA, M. LAZZARI y M. D. GLASCOCK, 2004. Quest for ancient routes: Obsidian sourcing research in Northwestern Argentina. Journal of Archaeological Science 31: 193-204. 